\title{
NEW DETERMINATIONS OF THE CHEMICAL COMPOSITION OF WOLF-RAYET STARS
}

\author{
T. NUGIS \\ Tartu Astrophysical Observatory, EE2444 Tõravere, Estonia
}

\begin{abstract}
The formation of the lines of ions $\mathrm{He}, \mathrm{H}, \mathrm{N}$ and $\mathrm{C}$ in the WR stars of different spectral subtypes has been studied using clumped wind models. Our new results agree well with the previous results obtained with smooth wind codes which make use of highly excited lines of different ions.
\end{abstract}

Key words: stars: Wolf-Rayet - clumped winds - abundances

\section{Clumped winds}

The density inhomogeneities (clumps) seem to have quite an important role in explaining the peculiar properties of WR spectra: 1) excess IR-fluxes as compared to the values expected from radio-fluxes; 2) IR eclipses in V444 Cyg; 3) weakness of electron scattering wings in emission lines; 4) excess densities obtained from standard models as compared with the values determined from radio-fluxes; 5) X-ray radiation. Antokhin et al. (1992) showed that clumped wind models yield the observed He II and He I line profiles with smaller mass loss rates and higher stellar temperatures as compared with smooth wind models. Therefore, the basic parameters of WR stars need reexamination. In the present communication we will present the results of chemical composition determinations of WR stars obtained by the use of clumped wind models. The clumps are assumed to originate at the effective stellar surface and are regarded to be conservative formations which are expanding with the mass-average speed $v_{c l}$ when moving away from the stellar surface: $v_{c l}=\frac{v_{s}}{\gamma} \sqrt{\frac{2 \gamma}{(\gamma-1)}}$, where $\gamma$ is the adiabatic exponent and $v_{s}$ is the adiabatic sound speed. The clumps are assumed to move according to the same velocity law as the interclump medium. For model computations we need to specify the filling factor, the contrast and the mean size of clumps at the stellar surface. At every other point in the envelope these parameters can be found from the equations given in Antokhin et al. (1992).

\section{H/He ratios}

$\mathrm{H} / \mathrm{He}$ ratios were determined for $30 \mathrm{WR}$ stars using the clumped wind code by Nugis \& Niedzielski (1994). Theoretical line fluxes of H I, He II and He I lines were found summing up the contributions from different layers of the wind. The level population statistical equilibria equations were solved together for He II and $\mathrm{H}$ I in the Sobolev approximation by taking into account 
the effects of the line overlap in the expanding medium. As all hydrogen lines are slightly redshifted with respect to blending He II lines, the line overlap effects must be taken into account. The level populations of He II are not influenced by $\mathrm{HI}_{\mathrm{I}}$. Our computations showed that the populations of $\mathrm{HI}_{\mathrm{I}}$ are not strongly influenced by the suppression of stellar continuum (due to absorption by blending He II atoms) and by line radiation of surrounding HeII atoms but line radiation of He II is strongly suppressed (especially in the Pickering series) due to absorption caused by $\mathrm{H}$ I atoms lying in front of He II atoms (in relation to a distant observer). The He II $k-6$ series lines are only slightly influenced by this effect and they are better suited for the determinination of the $\mathrm{H} / \mathrm{He}$ ratios. Our $\mathrm{H} / \mathrm{He}$ ratios agree quite well with the results obtained through the line profile studies by Hamann $e$ al al (1991) and by Crowther (1993). The $\mathrm{H} / \mathrm{He}$ ratio in WN stars is lower than the mean cosmic ratio and in WC stars no hydrogen seems to be present. Hydrogen abundance is decreasing when going from WN8-9 to WN3 stars. A substantial intrinsic scatter seems to be present among the stars of the same subtype.

\section{C, $\mathbf{N}$ abundances}

By comparing the clumped wind predicted line fluxes of highly excited lines of different ions of $\mathrm{C}, \mathrm{N}$ with the observed fluxes (the ratios of fluxes of these lines are not very sensitive to concrete details of different models), we determined the relative abundances of $\mathrm{N}$ and $\mathrm{C}$ for ten $\mathrm{WN}$ stars and five WC stars. Theoretical fluxes of the lines of $\mathrm{C}, \mathrm{N}$ ions were found by summing up the contributions from different layers of the wind. Our results of $\mathrm{N} / \mathrm{He}$ and $\mathrm{C} / \mathrm{He}$ ratios for $\mathrm{WN}$ stars $(\mathrm{N} / \mathrm{He} \approx 0.002-0.006, \mathrm{C} / \mathrm{N} \approx 0.05$ according to the number of atoms) agree well with the results obtained by Crowther (1993). The $\mathrm{C} / \mathrm{He}$ ratios determined for $\mathrm{WC}$ stars $(\mathrm{C} / \mathrm{He} \approx 0.1-0.5)$ agree quite well with the previous results (Nugis 1991). The $\mathrm{C} / \mathrm{He}$ ratios increase when going from late to early WC subtypes. WN stars exhibit material which has undergone hydrogen burning and WC stars show more advanced evolutionary stage with helium burning material peeled off.

\section{References}

Antokhin, I.I., Nugis, T., Cherepashchuk, A.M. 1992, Astron. Zh. 69, 516

Crowther, P.A. 1993, $\mathrm{PhD}$ Thesis, University of London

Hamann, W.-R., Dünnebeil, G., Koesterke, L., Schmutz, W., Wessolowski, U. 1991, $A \& A$ 249,443

Nugis, T. 1991, in: G. Michaud \& A. Tutukov (eds.), Evolution of Stars: the Photospheric Abundance Connection, Proc. IAU Symp. No. 145 (Dordrecht: Kluwer), p. 209

Nugis, T., Niedzielski, A. 1994, $A \mathscr{E} A$ submitted 